\title{
Le traducteur et son image. Étude des couvertures d'ouvrages de traductologie
}

\author{
The translator and his image. \\ A study of the covers of books on translation
}

\author{
Elżbieta Skibińska \\ Université de Wrocław, Wrocław, Pologne \\ elzbieta.skibinska@uwr.edu.pl
}

\begin{abstract}
The front cover of a book usually contains the title, the authors'names, the publisher's logo and an illustration. All these elements announce the content of the book, and the paraverbal elements (illustration, typographical arrangement...) can also be used to awaken the curiosity of the potential reader. This article deals with the reproductions of art works chosen by the editors for the front covers of Translation Studies books. Cover illustrations are treated here as a kind of "definition of translator's work through a pictorial metaphor", i.e. as a representation of how translation can be understood or as an indication of its important features. The analysis of these illustrations shows the various means used by the publishers of Translation Studies books to define translation through pictures.
\end{abstract}

Keywords: translator, representation, front cover, illustration, Translation Studies books 


\begin{abstract}
Dans tous les cas, sous quelque forme qu'elle se présente, la première de couverture mérite son titre de «première » si elle récapitule en l'unité d'une harmonie certaine, couleurs, lettres et symboles ; noms, titres et logo éditorial, le tout dans une maquette artistique qui accroche le regard, suscite la curiosité, met l'imagination en alerte et l'esprit en attente.
\end{abstract}

Sauvé (2006, p. 285)

\title{
INTRODUCTION
}

Cette étude tire son origine d'une expérience vécue lors d'un cours de traductologie pendant lequel la discussion tournait autour du passage suivant de Traduire. Théorèmes pour la traduction :

La finalité d'une traduction consiste à nous dispenser de la lecture du texte original - voilà les termes dans lesquels il convient selon nous de définir ce qu'est proprement une traduction. La traduction est censée remplacer le texte-source par le " même » texte en langue cible. C'est le caractère problématique de cette identité qui fait toute la difficulté d'une théorie de la traduction. On parlera d'« équivalence » (Ladmiral, 1979, p. 15).

Le « caractère problématique de cette identité » souligné par Jean-René Ladmiral s'est avéré, ce jour-là, particulièrement épineux pour les étudiants. L'idée m'était venue de recourir à l'image. L'un des tableaux de René Magritte, La Trahison des images (montrant une pipe accompagnée de sa légende « Ceci n'est pas une pipe »), projeté sur le grand écran de la salle, nous a servi de point de départ pour commenter l'emploi par Ladmiral des guillemets autour du mot même.

Depuis, les œuvres d'art - en particulier les tableaux - et leur usage possible en traductologie attirent mon attention. Rappelons que pendant longtemps, la peinture a servi de base à la construction d'un langage approprié pour parler de la traduction. Les $\mathrm{XVII}^{\mathrm{e}}$ et XVIII ${ }^{\mathrm{e}}$ siècles ont vu la formation de nombreuses comparaisons ou métaphores qui véhiculaient une conception de la traduction perçue comme imitation, et qui insistaient sur l'analogie entre le travail du traducteur et celui du copiste (D'hulst, 1993). Ce passage de l'article de Marmontel en offre un exemple éloquent :

On a dit de la traduction qu'elle était comme l'envers de la tapisserie : cela suppose une industrie bien grossière et bien maladroite. Faisons plus d'honneur au copiste, et accordons-lui en même temps l'adresse de bien saisir le trait et de bien placer les couleurs : s'il a le même assortiment de nuances que l'artiste original, il fera une copie exacte, à laquelle on ne désirera que le premier feu du génie ; mais s'il manque de demi-teintes, ou s'il ne sait pas les former du mélange de ses couleurs, il ne donnera qu'une esquisse, d'autant plus éloignée de la beauté du tableau, que celui-ci sera mieux peint et plus fini (Jean-François Marmontel, cité dans D’hulst, 1990, p. 54-55).

On peut cependant envisager autrement la relation entre la peinture et la traduction : certains tableaux peuvent être traités comme une « définition par métaphore » 
de la traduction. Et on peut considérer comme lieu privilégié de la manifestation de cette métaphore les couvertures des ouvrages de traductologie : les reproductions de peintures qui y apparaissent sont sciemment employées pour véhiculer une certaine façon de comprendre ou représenter la traduction.

Afin de vérifier cette hypothèse, $\mathrm{j}$ 'ai entrepris d'étudier les premières de couverture des ouvrages de traductologie, ouvrages individuels ou collectifs sous la direction d'un chercheur, publiés dans différents pays et langues, majoritairement par des maisons d'éditions spécialisées : éditeurs scientifiques et universitaires (Universitas, LIT Verlag, Routledge, Benjamins, Wydawnictwo Uniwersytetu Jagiellońskiego, Artois Presses Université, Septentrion...) ou dans des collections spécialisées (Routledge Advances in Translation and Interpreting Studies, Benjamins Translation Library, Translatio, Vita Traductiva...). Parmi les centaines de livres publiés, j'ai examiné ceux auxquels j'ai pu accéder directement, ce qui représente cent neuf couvertures, dont trente-six contiennent une reproduction d'œuvre d'art.

J'ai exclu de l'analyse celles où le tableau reprend le message linguistique du titre, par ex. la couverture de Traduire en langue française en 1830, illustrée par un tableau d'Eugène Delacroix, La liberté guidant le peuple, qui renvoie aux « Trois Glorieuses » de juillet 1830, ou celle de l'ouvrage publié sous la direction de Jean Delisle, Portraits de traducteurs, reproduisant l'œuvre de Gu Gongdu Un traducteur. Finalement, l'ensemble ainsi constitué comprend vingt-six couvertures qui peuvent être considérées comme support d'une métaphore picturale de la traduction, dans le sens défini plus loin (voir 1.3). Cinq parmi celles-ci ont en commun de montrer des personnages occupés à une activité qui - selon le concepteur de la couverture - présenterait des traits communs avec le travail du traducteur. En effet, c'est celui-ci qui m'intéresse ici'.

\section{PRÉLIMINAIRES : LA COUVERTURE DU LIVRE}

1.1. Depuis la parution de Seuils de Gérard Genette en 1987, on traite la couverture du livre comme un paratexte : une de ces « productions, elles-mêmes verbales ou non, comme un nom d'auteur, un titre, une préface, des illustrations [...]» qui entourent et prolongent le texte « pour le présenter, au sens habituel de ce verbe, mais aussi en son sens le plus fort : pour le rendre présent, pour assurer sa présence au monde, sous la forme [...] d'un livre » (Genette, 1987b, p. 7). En plus de trente ans, ces « bagatelles de la porte» (Genette, 1987a, p. 3) ont fait l'objet de nombreuses études, et sont bien mieux connues.

${ }^{1}$ Dans une étude précédente, je m'étais concentrée sur les couvertures qui « montrent » la relation entre le texte original et le texte traduit (Skibińska, 2021). 
La couverture - «bagatelle de la porte » par excellence - suscite un intérêt croissant des chercheurs de différentes disciplines: bibliologues, sociologues des médias, sémioticiens, comparatistes... (Szczęśniak, 2011 ; Hojka, 2012 ; Lachman, 2012). Son étude relève aussi des neurosciences cognitives qui explorent le phénomène de la créativité, et de la traductologie, si l'on considère la couverture comme une traduction intersémiotique (Sonzogni, 2011 ; Mossop, 2018). L'attention des chercheurs porte aussi sur l'aspect « commercial » du livre en tant que produit, leurs travaux insistant sur les mécanismes de marketing et sur le rôle des péritextes éditoriaux qui interviennent dans les stratégies de marché (Baillet, 2010 ; Narbutowicz, 2012).

Cette multiplicité des approches vient de la situation de communication particulière dans laquelle se situe la couverture, et de sa dimension pragmatique : « La couverture assure une fonction importante de présentation et d'incitation à l'achat car elle est (presque) automatiquement regardée par l'acheteur (ou l'emprunteur) qui manipule le livre » (Lane, 1992, p. 19). En effet, si l'éditeur, comme tout « producteur culturel », est un personnage double, le livre, effet de son activité, est un objet double, une « réalité à double face, marchandise et significations » (Bourdieu, 1966, p. 870).

C'est sur la « face significations », pour utiliser la distinction faite par Bourdieu, que nous allons nous concentrer. En effet, si les deux faces ne peuvent pas être séparées l'une de l'autre, l'importance de la « face marchandise » semble moindre dans le cas des ouvrages de recherche. Ils ne sont pas un objet de consommation au même titre que les œuvres littéraires. Pour le chercheur intéressé par les travaux en relation avec sa recherche, ils sont une voie d'accès à des connaissances. Ce n'est donc pas l'illustration de la couverture qui est le facteur décisif d'incitation à la lecture.

Elle peut néanmoins attirer l'attention par sa façon d'annoncer le contenu de l'ouvrage en se conjuguant à l'élément verbal (le titre). « [L'illustration] véhicule également certaines significations auxquelles les consommateurs ne sont pas indifférents ", constate Baillet (2010, p. 148). Si l'auteure parle des romans, sa constatation peut s'appliquer aussi aux ouvrages de recherche, avec cette précision que le « consommateur » d'un tel ouvrage est généralement un spécialiste, qui aborde le livre avec des connaissances préalables lui permettant de situer la collection, l'auteur, le titre dans un courant de la pensée traductologique.

1.2. Les analyses qui vont suivre s'appuient sur les prémisses suivantes :

- la couverture constitue un message hybride, verbal (linguistique) et iconique ;

- elle peut être traitée comme un message autonome, telle une affiche, sans nécessité de lui chercher une motivation dans le contenu du livre ; mais, en même temps, elle est en lien avec celui-ci, puisqu'elle est censée le présenter en en offrant une sorte de " sommaire iconique », ou encore une « traduction intersémiotique » (Sonzogni, 2011, p. 5) ; cette présentation a pour but de diriger ou d'influencer la lecture ; il se crée ainsi une interaction entre « l'extérieur » et «l'intérieur» du livre ; comparée à la situation d'un roman, celle 
des ouvrages de recherche est cependant plus compliquée : si dans de nombreux cas il s'agit du travail d'un seul auteur, donc d'un tout homogène dont l'essentiel peut être contenu dans un résumé, les livres qui nous intéressent ici sont souvent des publications collectives, aux contenus hétérogènes et parfois même contradictoires ; la présentation véhiculée par la couverture est censée, dans ce cas, signaler ce qui est commun aux contributions (la traduction comme objet de réflexion);

- la couverture est le résultat de la rencontre (consensus ou compromis) des intentions de l'auteur ou directeur de l'ouvrage, des attentes du lecteur potentiel, des stratégies de l'éditeur et de ses possibilités matérielles, des traditions, des modes ou des tendances du marché (local et global), et - last but not least - des talents du maquettiste.

Considérant le caractère hybride de la première de couverture, je me limite à la relation titre (texte; message verbal) - illustration (image; message iconique).

1.3. Selon Roland Barthes, le message linguistique peut avoir deux fonctions par rapport au message iconique : celle d'ancrage et celle de relais. La première consiste à donner une certitude quant à la lecture de l'image, la deuxième souligne l'« entrelacement » qui fait que les deux messages deviennent nécessaires l'un à l'autre :

Au niveau du message littéral, la parole répond, d'une façon plus ou moins directe, plus ou moins partielle, à la question : qu'est-ce que c'est ? Elle aide à identifier purement et simplement les éléments de la scène et la scène elle-même : il s'agit d'une description dénotée de l'image (description souvent partielle) [...] Au niveau du message « symbolique », le message linguistique guide non plus l'identification, mais l'interprétation, il constitue une sorte d'étau qui empêche les sens connotés de proliférer [...]. La fonction de relais est plus rare (du moins en ce qui concerne l'image fixe) ; on la trouve surtout dans les dessins humoristiques et les bandes dessinées. Ici la parole (le plus souvent un morceau de dialogue) et l'image sont dans un rapport complémentaire ; les paroles sont alors des fragments d'un syntagme plus général, au même titre que les images, et l'unité du message se fait à un niveau supérieur : celui de l'histoire, de l'anecdote, de la diégèse [...] (pp. 44-45).

Cette proposition de Barthes a été appliquée par Baillet à la relation entre l'illustration et le titre :

En effet, comme son nom l'indique, l'illustration illustre le roman et notamment son titre. C'est donc elle qui suppléait [sic!] les carences expressives du titre par le biais d'une image plus ou moins narrative mais dont le but est d'apporter aux consommateurs des informations sur le contenu, ou du moins, sur l'atmosphère du roman (p. 150).

Dans le cas des couvertures qui nous intéressent ici, on peut envisager une autre situation encore. Toutes comprennent, dans le titre, le mot traduction (translation, przekład, traduzzione...) ou un dérivé comme traducteur, traduire... ; en annonçant 
la thématique de l'ouvrage, ce mot constitue un ancrage pour l'illustration et en guide l'interprétation. L'élément iconique remplit, quant à lui, la fonction de relais, en complétant, de façon économique, le message verbal auquel il apporte ce que celui-ci ne peut pas communiquer.

Cependant, la traduction est un concept qui échappe à une définition unique ${ }^{2}$, et encore plus à une représentation iconique réaliste. L'illustration juxtaposée au titre ne montre donc pas la traduction elle-même, en tant que telle, mais propose une « scène » qui partage avec la traduction une qualité essentielle. En d'autres termes, le tableau reproduit sur la couverture représente un élément de la réalité qui, selon l'auteur de la couverture, serait à son tour représentatif du travail du traducteur.

Cette analogie permet de traiter la rencontre de deux éléments formellement et sémantiquement disparates comme un message métaphorique, basé sur une « métaphore créative », telle que l'a définie Max Black. Rappelons qu'il a rejeté le principe de la ressemblance de deux réalités comme condition de création de la métaphore ; au contraire, selon lui, c'est la métaphore qui crée cette ressemblance, ou analogie, en « s'imposant » à son auteur dans un « flash d'intuition ». Il a souligné aussi le caractère innovant de la pensée métaphorique et de l'expression par métaphore, qui permettent de saisir le monde d'une manière qui dépasse les limites du langage conventionnel (Black, 1979).

L'idée de la métaphore traitée comme un phénomène cognitif, et pas seulement comme un procédé linguistique (poétique), est à la base de la théorie cognitiviste de George Lakoff et Mark Johnson développée à la même époque. Pour eux, la fonction première de la métaphore est de permettre de comprendre, ne serait-ce que partiellement, une expérience par le biais d'une autre expérience (provide a partial understanding of one kind of experience in terms of another experience) (Lakoff and Johnson, 1980, p. 154). Les deux expériences peuvent être liées par une ressemblance antérieure ou « objective », mais leur rapprochement peut en créer de nouvelles. Ces auteurs insistent sur le fait que la métaphore relève d'abord d'un mode de pensée ; elle est avant tout un outil cognitif qui offre un moyen économique de transmettre des significations. Cette idée ouvre la voie à la réflexion sur la possibilité d'appliquer le concept de métaphore à d'autres médias (Forceville, 1996 ; Ventalon, Domínguez Mayo, Tijus, Escalona Cuaresma, 2017).

Dans la suite, la couverture à reproduction d'œuvre picturale sera considérée comme un message hybride (verbal et iconique) de caractère métaphorique : en puisant dans sa connaissance du monde, son concepteur crée une similitude entre le phé-

\footnotetext{
${ }^{2}$ « Je dirai que, paradoxalement, le concept de traduction est lui-même inassignable. Tout le monde sait ce que c'est qu'une traduction. Mais dès lors qu'on entreprend de définir le terme, on est un peu comme Heidegger, au tout début de Sein und Zeit, qui (citant le Sophiste de Platon) relève que tous font un usage courant du verbe "être" (du mot "étant") mais que, dès qu'on veut s'assurer de ce qu'on entend par là, on se retrouve "dans l'embarras" (in Verlegenheit). Je dirai que mutatis mutandis c'est la même chose pour la traduction » (Ladmiral, 2004, par. 32).
} 
nomène de la traduction et un autre phénomène, celui qui se trouve représenté dans l'œuvre reproduite (la « scène »). Celle-ci a généralement un titre, mais puisqu'il est absent de la couverture, il perd sa fonction de guide de lecture de l'œuvre. En outre, le titre du livre - l'élément verbal - ne sert pas à identifier la « scène » dépeinte dans l'œuvre ; en lui donnant un nouveau « nom », il suscite une nouvelle interprétation visant à dégager ce que la "s scène » et la problématique annoncée dans le titre ont en commun, et à construire ainsi le sens du message hybride véhiculé par la couverture.

La lecture des tableaux est forcément simplifiée, car je me limite à la recherche d'une ressemblance entre le travail du traducteur et la scène représentée, donc à ce que je considère comme essentiel pour la reconstruction de la métaphore de la traduction.

\section{CE QUE LES PREMIÈRES DE COUVERTURE NOUS APPRENNENT SUR LE TRADUCTEUR ET SON TRAVAIL}

Trois des couvertures analysées contiennent une reproduction d'œuvre qui évoque l'idée de juste valeur ou d'égalité des valeurs. La première est Le prêteur et sa femme, appelé aussi Le Changeur et sa femme ou Le Banquier et sa femme, tableau de Quentin Metsys ${ }^{3}$ figurant sur la couverture de L'Épreuve de l'étranger. Culture et traduction dans l'Allemagne romantique d'Antoine Berman (1984)4. Quel que soit le métier du personnage, l'élément primordial de la scène est la pesée des pièces d'or à l'aide d'une balance de précision. Le but de l'opération est d'en déterminer la valeur pour procéder ensuite à un échange avec le client que l'on aperçoit dans le petit miroir. Il s'agit donc d'établir une équivalence de valeur des biens échangés : des pièces contre des pièces? des pièces contre des perles? Cela n'a pas d'importance, ce qui importe, c'est de les mettre en rapport d'équivalence.

Avec le mot équivalence, on entre sur le terrain de la traductologie où, depuis Jakobson, il est devenu un " incontournable », surtout dans le courant structuraliste des années 60 et $70 \mathrm{du} \mathrm{XX}^{\mathrm{e}}$ siècle. Il renvoie à l'idée que le TO et le TT partagent « quelque chose » qui a la même valeur. Mais le « quelque chose » étant extrêmement difficile à saisir et à définir, le terme - ou plutôt la notion à laquelle il renvoie - a suscité tant d'interprétations que son usage a presque failli être abandonné (Pym, 1995). Et pourtant, comme le remarque Brian Mossop :

[...] equivalence is such an important idea: it's the theory of translation implicitly held by the average person, and thus the average paying client and the average reader of translations. Since readers and clients are key participants in translation, their beliefs should

${ }^{3}$ https://fr.wikipedia.org/wiki/Le_Pr\%C3\%AAteur_et_sa_femme\#/media/File:Quentin_Massys_001.jpg

${ }^{4}$ http://www.gallimard.fr/Catalogue/GALLIMARD/Tel/L-Epreuve-de-1-etranger 
presumably be a part of translation theory, and thus a part of the mental framework of translators as they select their TL wordings (Mossop).

C'est précisément à cette conception doxique du travail du traducteur - recherche de l'équivalence - que renvoie en premier lieu, je pense, le tableau de Metsys reproduit sur cette couverture 5 .

Établir une équivalence suppose aussi de l'honnêteté, de la confiance et de la justesse, c'est-à-dire une dimension éthique, si importante dans la réflexion de Berman sur les relations entre les langues et les cultures qui se rencontrent dans l'acte de traduire. La question de l'éthique est posée expressis verbis dans le titre de l'ouvrage d'Anthony Pym Pour une éthique du traducteur (1997) ${ }^{6}$. Sa couverture mérite une attention particulière : le tableau choisi pour l'illustrer, The Money Changers, est attribué à un peintre anonyme, imitateur de Marinus Van Reymerswaele, un peintre néerlandais du $\mathrm{XVI}^{\mathrm{e}}$ siècle qui s'est lui même inspiré de l'œuvre de Quentin Metsys ${ }^{7}$. Il s'établit ainsi entre les deux couvertures un dialogue « interpictural» qui n'est pas anodin. En effet, l'auteur du livre - qui prône une attitude du traducteur basée sur le « respect de l'autre » et « la limitation de la souffrance » (Pym, 1997, p. 137) - mène une discussion avec Berman, en insistant sur la nécessité de centrer l'attention sur le traducteur (notamment le traducteur professionnel) et son rôle de « communicateur interculturel », et non pas sur la traduction, opération linguistique telle que la comprend l'auteur de L'Épreuve de l'étranger. Le tableau, qui est une sorte de réponse à celui de Metsys, sert de relais au mot éthique, en annonçant en même temps une discussion avec Berman.

Le trébuchet - " outil de l'équivalence » - apparaît aussi au centre du tableau La Femme à la balance (appelé également La Peseuse de perles, ou encore La Peseuse d'or) du peintre néerlandais Johannes Vermeer, reproduit sur la couverture du livre La Médiation de l'étranger. Une sociolinguistique de la traduction, de Jean Peeters $(1999)^{8}$. Si la première partie du titre fait écho à celui du livre de Berman (L'épreuve de l'étranger), la seconde souligne que la traduction est une activité fondamentalement sociale.

\footnotetext{
${ }^{5}$ Signalons au passage que l'image de la balance comme outil servant à établir l'équivalence des mots remonte à Cicéron : «J'ai mis en latin les deux plus célèbres discours des deux Attiques les plus éloquents, Eschine et Démosthène, discours dont l'un répond à l'autre ; je les ai mis en latin, non pas en traducteur mais en orateur ; les pensées restent les mêmes, ainsi que leur tour et comme leurs figures ; les mots sont conformes à l'usage de notre langue. Je n'ai pas cru nécessaire de rendre mot pour mot; c'est le ton et la valeur des expressions dans leur ensemble que j'ai gardés. J'ai cru qu'il me fallait payer le lecteur non pas en comptant pièce par pièce, mais pour ainsi dire en pesant la somme en bloc » (Cicéron, 1921 ; cité par Ballard, 1992, pp. 39-40).

${ }^{6} \mathrm{http}: / /$ apu.univ-artois.fr/Revues-et-collections/Traductologie/Pour-une-ethique-du-traducteur

${ }^{7}$ https://commons.wikimedia.org/wiki/File:Marinus_van_Reymerswaele_(Follower_of)_-_The Money_Changers_-_Google_Art_Project.jpg

${ }^{8} \mathrm{http}$ ://apu.univ-artois.fr/Revues-et-collections/Traductologie/La-Mediation-de-1-etranger.-Une-sociolinguistique-de-la-traduction
} 
Cependant, dans ce tableau qui ne représente qu'une seule personne - une femme en train de peser des éléments de valeur - la médiation semble absente. C'est le titre du livre qui suggère que la pesée et la recherche de l'exactitude peuvent déboucher sur l'établissement d'une mesure commune, d'un équilibre, d'un accord, d'une conciliation ou d'une entente. Si l'on considère les apports théoriques de Peeters comme une prise de position par rapport aux propositions de Berman et de Pym, on peut conclure que la recherche de l'équivalence symbolisée par le trébuchet et la pesée est le but primordial du travail du traducteur, que ce soit dans une optique culturelle, sociale ou éthique.

Conciliation, concorde, entente et égalité comme résultat du travail du traducteur se voient soulignées par le maquettiste du livre Traduire l'Europe, sous la direction de Françoise Barret-Ducrocq (1992) $)^{9}$, qui a eu recours à Pablo Picasso et sa Ronde de la jeunesse (1961). Les couleurs et les mouvements des personnages dansant autour d'une colombe qui tient en son bec un rameau d'olivier traduisent la joie et le sens de l'union, de l'accord et de l'harmonie. La traduction - « la langue de l'Europe ", selon l'expression attribuée à Umberto Eco - est ainsi présentée comme une activité extrêmement positive, permettant la communication qui mène à la communion.

La couverture du livre d'Emily Apter The Translation Zone (2006 ${ }^{10}$; traduction française 2015) fait penser, elle aussi, aux effets du travail des traducteurs (des interprètes, en l'occurrence). Elle reproduit une photo utilisée par l'artiste catalan Antoni Muntadas dans son installation On Translation. The Games (1996). Celle-ci appartient à un cycle d'installations dans lesquelles, en se servant de divers moyens techniques (vidéo, sites Internet, logiciels de TAO ou « traducteurs automatiques » comme Babelfish), Muntadas attire l'attention sur l'omniprésence de la traduction comprise comme un médium ou un filtre. La photo reproduite sur la couverture du livre d'Apter représente des destinataires du travail des traducteurs : des diplomates, représentants de divers pays lors d'un congrès international... Mais elle dévoile indirectement l'existence des interprètes qui, invisibles, enfermés dans leur cabines insonorisées, transmettent, en les filtrant, les informations médiées qui parviennent dans les écouteurs de l'auditoire. Au-delà, elle fait prendre conscience du rôle de la traduction aujourd'hui et de l'importance des traducteurs dans le monde globalisé ${ }^{11}$.

\footnotetext{
${ }^{9} \mathrm{https} / /$ www.abebooks.fr/rechercher-livre/titre/traduire-1\%60europe/auteur/barret-ducrocq-francoise/

${ }^{10} \mathrm{https} / /$ press.princeton.edu/books/paperback/9780691049977/the-translation-zone

${ }^{11}$ L'installation, dont la photo n'est qu'un élément, s'inscrit aussi dans un ensemble d'autres réalisations qui prennent le traducteur pour objet de représentation artistique : romans, films, et même bandes dessinées (cf. Gambier, 2012). Elle peut être considérée aussi comme exemple du traitement de la traduction perçue comme un phénomène offrant des moyens utilisés dans l'art pour comprendre ou commenter la complexité d'un monde en mutation.
} 


\section{CONCLUSION}

L'œuvre picturale reproduite sur la couverture d'un ouvrage de traductologie sertt-elle à véhiculer une certaine image du traducteur et de son travail? Cette première « reconnaissance du terrain », qui s'appuie sur un échantillon certes limité, montre déjà que la réponse est affirmative. Les cinq couvertures soumises à l'étude présentent le traducteur comme un médiateur ou intermédiaire guidé en principe par une éthique d'égalité ; son apport peut avoir des conséquences graves pour les individus, mais aussi pour des groupes (sociétés, nations, cultures...).

L'exploration d'un corpus plus étendu pourrait porter sur un éventail de questions plus large, qui iraient au delà de celle concernant l'image du traducteur. J'en signale quelques-unes qui - lors de la sélection des couvertures à analyser - m'ont paru particulièrement prometteuses.

La première porte sur le rôle des titres des œuvres reproduites. Dans cette étude, ils ont été cités pour identifier l'œuvre, mais leur sens n'a pas été pris en considération; or, d'une part, il oriente la lecture du tableau, et de l'autre, il peut influencer la lecture du titre de l'ouvrage et induire un jeu avec lui. Par ailleurs, parmi les œuvres examinées, aucune ne s'intitule Traducteur. Cela ne veut pas dire que de telles œuvres n'existent pas $^{12}$; mais la relation message verbal-message iconique est, dans ces cas, différente de celle de la métaphore qui m'intéresse ici.

La deuxième concerne la récurrence de certaines notions (telles 'équivalence', 'médiation' 'éthique') qui apparaissent lors de l'analyse des couvertures. Leur recensement et leur fréquence d'apparition pourraient nous éclairer sur la circulation (ou la pérennité) de certaines conceptions de la traduction.

La troisième se rapporte au processus de décision qui sous-tend le choix de l'œuvre à reproduire sur la couverture. Des configurations variées, liées à l'engagement de l'auteur et/ou du graphiste, aux relations de l'auteur et/ou de l'éditeur avec l'artiste, à la possibilité d'acquérir les droits de reproduction, etc., sont possibles. Une étude basée sur une enquête auprès de différents agents du processus éditorial pourrait informer sur les mécanismes qui décident de la représentation de la traduction véhiculée sur les couvertures.

Mon recours à la peinture pour « représenter » la traduction à mes étudiants, évoqué au début de cette étude, reposait sur une intuition didactique. L'examen du message hybride, verbal et iconique, véhiculé par les couvertures des travaux de traductologie a dévoilé des intentionnalités qui méritent d'être encore étudiées.

${ }^{12} \mathrm{Au}$ contraire - et l'on peut citer comme exemple, le tableau de Wilhelm Sasnal Shoah (Ttumaczka) [Shoah (Traductrice)] reproduit sur la couverture du livre de Małgorzata Tryuk « Ty nic nie mów, ja będę thumaczył ». O etyce w thumaczeniu ustnym [« Ne dis rien, je vais traduire ». Sur l'éthique de l'interprète], qui mériterait une étude à part. 


\section{BIBLIOGRAPHIE}

Ouvrages analysés :

Apter, E. (2006). The Translation Zone: A New Comparative Literature. Princeton : Princeton University Press. Apter, E. (2015). Zones de traduction : pour une nouvelle littérature comparée (traduit de l'anglais par Hélène Quiniou). Paris : Fayard.

Barret-Ducrocq, F. (ed.) (1992). Traduire l'Europe. Paris : Payot.

Berman, A. (1984). L'Épreuve de l'étranger. Culture et traduction dans l'Allemagne romantique. Paris : Gallimard.

Peeters, J. (1999). La Médiation de l'étranger. Une sociolinguistique de la traduction. Arras : Artois Presses Université.

Pym, A. (1997). Pour une éthique du traducteur. Arras : Artois Presses Université, Presses de l'Université d'Ottawa.

Ouvrages cités :

Ballard, M. (1992). De Cicéron à Benjamin. Traducteurs, traductions, réflexions. Lille : Presses Universitaires de Lille.

Baillet, C. (2010). Le choix d'un roman sur le point de vente. Influence des variables situationnelles et des caractéristiques du consommateur sur le processus décisionnel utilisé. Sarrebrueck : Editions Universitaires Européennes.

Barthes, R. (1964). Rhétorique de l'image. Communications, 4, 40-51, doi.org/10.3406/comm.1964.1027.

Black, M. (1979). More about Metaphor. In A. Ortony (ed.), Metaphor and Thought (pp. 19-43). Cambridge et al.: Cambridge University Press, doi.org/10.1017/CBO9781139173865.004.

Bourdieu, P. (1966). Champ intellectuel et projet créateur. Les Temps modernes, 246, 865-906.

D'hulst, L. (1990). Cent ans de théorie française de la traduction. De Boiteux à Littré (1748-1847). Lille : Presses Universitaires de Lille.

D'hulst, L. (1993). Observations sur l'expression figurée en traductologie française (XVIIIe-XIXe siècles). TTR : traduction, terminologie, rédaction, 6 (1), 83-111, doi.org/10.7202/037139ar.

Forceville, Ch. (1996). Pictorial Metaphor in Advertising. London and New York : Routledge, doi. org/10.4324/9780203272305.

Gambier, Y. (2012). Le traducteur défiguré ? Romanica Wratislaviensia, 59, 13-24.

Genette, G. (1987a). Présentation. Poétique, 69.

Genette, G. (1987b). Seuils. Paris : Le Seuil.

Hojka, B. (2012). Okładka książkowa z perspektywy. In M. Komza (ed.), W poszukiwaniu odpowiedniej formy: rola wydawcy, typografa, artysty i technologii w pracy nad książka (pp. 61-72). Wrocław : Wydawnictwo Uniwersytetu Wrocławskiego.

Lachman, M. (2012). Okładkowy stan posiadania (w literaturze najnowszej). Teksty Drugie, 6, 1-117.

Ladmiral, J.-R. (1979). Traduire. Théorèmes pour la traduction. Paris : Payot.

Ladmiral, J.-R. (2004). Lever de rideau théorique : quelques esquisses conceptuelles. Palimpsestes, 16, 15-30, doi.org/10.4000/palimpsestes. 1587.

Lane, P. (1992). La périphérie du texte. Paris : Nathan.

Mossop, B. (2010). Review of Anthony Pym, "Exploring Translation Theories », Routledge 2010, consulté le 15.04.2021 sur : http://www.yorku.ca/brmossop/PymExploring.htm.

Mossop, B. (2018). Judging a translation by its cover. The Translator, 24, 1, 1-16. DOI : 10.1080/13556509.2017.1287545.

Narbutowicz, P. (2012). Sprzedać książkę po okładce. Warszawa : Biblioteka Analiz.

Pym, A. (1995). European Translation Studies, Une science qui dérange, and Why Equivalence Needn't Be a Dirty Word. TTR : traduction, terminologie, rédaction, 8 (1),153-176, doi.org/10.7202/037200ar. 
Sauvé, M. (2006). Qu'est-ce qu'un livre? : de la page blanche à l'achevé d'imprimer. Paris : Les Editions Fides.

Skibińska, E. (2021). Obrazy, które „mówią”, czym jest tłumaczenie. O ilustracjach na okładkach książek przekładoznawczych. Teksty Drugie.

Sonzogni, M. (2011). Recovered Rose. A Study of Book Cover Design as Intersemiotic Translation. Amsterdam/Philadelphia : John Benjamins, doi.org/10.1075/z.169.

Szczęśniak, K. (2011). Okładka i obwoluta książki jako przedmiot badań interdyscyplinarnych. Toruńskie Studia Bibliologiczne, 2, 29-41, doi.org/10.12775/TSB.2011.015.

Ventalon, G., Domínguez-Mayo, F.J., Tijus, Ch., Escalona Cuaresma, M.J. (2017). Études théoriques et empiriques sur la métaphore dans l'image : une revue de la littérature. L'Année psychologique, 117, 467-488, doi.org/10.4074/S000350331700402X. 